\title{
Diverting attention suppresses human amygdala responses to faces
}

\author{
Carmen Morawetz ${ }^{1,2,3,4}{ }^{*}$, Juergen Baudewig ${ }^{1,3,4}$, Stefan Treue ${ }^{2,5}$ and Peter Dechent ${ }^{1}$ \\ MR-Research in Neurology and Psychiatry, Georg-August University Goettingen, Goettingen, Germany \\ 2 Cognitive Neuroscience Laboratory, German Primate Center, Goettingen, Germany \\ ${ }^{3}$ Department of Education and Psychology, Freie Universitaet Berlin, Berlin, Germany \\ ${ }^{4}$ Cluster of Excellence "Languages of Emotion," Freie Universitaet Berlin, Berlin, Germany \\ ${ }^{5}$ Bernstein Center of Computational Neuroscience Goettingen, Goettingen, Germany
}

\section{Edited by:}

Shuhei Yamaguchi, Shimane University, Japan

\section{Reviewed by:}

Antonio Rangel, CalTech, USA

Alain Dagher, Montreal Neurological

Institute and Hospital, Canada

${ }^{*}$ Correspondence:

Carmen Morawetz, Cluster of

Excellence: Languages of Emotion,

Freie Universitaet Berlin,

Habelschwerdter Allee 45, 14195

Berlin, Germany.

e-mail:carmen.morawetz@fu-berlin.de
Recent neuroimaging studies disagree as to whether the processing of emotion-laden visual stimuli is dependent upon the availability of attentional resources or entirely capacity-free. Two main factors have been proposed to be responsible for the discrepancies: the differences in the perceptual attentional demands of the tasks used to divert attentional resources from emotional stimuli and the spatial location of the affective stimuli in the visual field. To date, no neuroimaging report addressed these two issues in the same set of subjects. Therefore, the aim of the study was to investigate the effects of high and low attentional load as well as different stimulus locations on face processing in the amygdala using functional magnetic resonance imaging to provide further evidence for one of the two opposing theories. We were able for the first time to directly test the interaction of attentional load and spatial location. The results revealed a strong attenuation of amygdala activity when the attentional load was high. The eccentricity of the emotional stimuli did not affect responses in the amygdala and no interaction effect between attentional load and spatial location was found. We conclude that the processing of emotional stimuli in the amygdala is strongly dependent on the availability of attentional resources without a preferred processing of stimuli presented in the periphery and provide firm evidence for the concept of the attentional load theory of emotional processing in the amygdala.

Keywords: attention, cognitive modulation, emotion, fMRI, fusiform gyrus, human brain

\section{INTRODUCTION}

Selective attention devotes cognitive resources to behavioral relevant stimuli and events, thereby enhancing the processing of attended relative to unattended information (Desimone and Duncan, 1995; Treue, 2003). A primary way to determine the importance of a stimulus or event is to evaluate its emotional significance (Compton, 2003). Emotionally significant stimuli receive enhanced processing via two different operating attentional mechanisms: The first one evaluates emotional significance preattentively ("automatically"), whereas the second one gives these significant stimuli priority in the allocation of attentional resources. The amygdala has been identified to play a crucial role in the processing of emotional stimuli (Phan et al., 2002; Zald, 2003; Sergerie et al., 2008). In theoretical works it has been proposed that the amygdala generates a coarse, rapid, and automatic response to emotional stimuli based on inputs from a subcortical pathway via the superior colliculus and the pulvinar nucleus of the thalamus, whereas a fine-grained perceptual representation of emotion-laden stimuli involves the occipital and temporal visual neocortex (LeDoux et al., 1984; LeDoux, 1996; Adolphs, 2002). Through reciprocal connections to sensory cortical processing regions, such as the visual cortex (Amaral et al., 2003), the amygdala may mediate the facilitation of attentional and perceptual processes (Morris et al., 1998a; Anderson and Phelps, 2001; Vuilleumier et al., 2004; Phelps and LeDoux, 2005; Phelps, 2006). Although a great number of studies support the notion that some aspects of emotional meaning can be encoded very rapidly and without conscious awareness (Morris et al., 1998b, 1999; Whalen et al., 1998; Öhman, 2002, 2005; Killgore and Yurgelun-Todd, 2004; Liddell et al., 2005; Öhman et al., 2007), these studies do not directly address whether such processing demands attentional resources. Recent neuroimaging studies presented conflicting evidence on this issue.

The view that emotional stimuli are processed preattentively and independent of the availability of attentional resources, has been supported by several studies demonstrating amygdala responses to fearful faces under conditions of inattention (Vuilleumier et al., 2001; Anderson et al., 2003; Williams et al., 2005). The influence of emotional expression and that of selective attention on face processing, was investigated by manipulating the focus of attention while keeping the perceptual load identical across all conditions. As the amygdala was activated by fearful faces regardless of the initial allocation of spatial attention, these neuroimaging studies seem to suggest that fear-related responses mediated by the amygdala may be obligatory and not dependent upon the availability of attentional resources.

However, such findings do not necessarily imply that these emotional effects would be entirely immune to any task-related attentional influences, since increasing perceptual load (Lavie, 1995) or the presence of distractors (Kastner and Ungerleider, 2000) might reduce the degree of amygdala responses to emotion-laden stimuli. 
According to the attentional load theory (Lavie, 1995, 2005), when a task at hand places very high demands on the perceptual system in order to process task-relevant stimuli, the processing of taskirrelevant stimuli would be inhibited. On the other side, in situations of low perceptual load, any capacity not taken up in perception of task-relevant stimuli would "spill over" to the processing of taskirrelevant stimuli. In other words, the processing of task-irrelevant information is dependent upon the perceptual attentional load in such a way that irrelevant information processing only takes place under low attentional load conditions and is abolished by high attentional load. In line with this theory, Pessoa et al. (2002, 2005) and Pessoa (2005) have argued that the perceptual load of the current task influences the ability of the visual system to generate an internal representation and an enhanced processing of emotional stimuli. Accordingly, a growing number of studies indicate a resource-dependence of face encoding processes, using tasks of different attentional load to show that the activation of the amygdala is reduced during conditions of high perceptual load (Pessoa et al., 2002, 2005; Bishop et al., 2007; Mitchell et al., 2007). An example is a recent study by Silvert et al. (2007) investigating whether the processing of peripheral face stimuli can be influenced by attention under different perceptual loads exerted by a competing task. In this paradigm differences in amygdala responses to fearful versus neutral unattended faces vanished during the high-load task, whereas in the low-load task a significant interaction between emotion and task was obtained. However, it is important to note that even during the highload task amygdala responses to unattended fearful and neutral faces were not completely suppressed, suggesting that not all attentional resources had been distracted by the competing task.

The strength of neural responses evoked by emotion-laden stimuli, especially in the amygdala, depends on numerous factors. One factor that could potentially account for the discrepant imaging results might be the spatial location of the presented emotional stimuli. The studies mentioned above support the notion that peripherally presented unattended fearful faces may activate the amygdala under both low (Vuilleumier et al., 2001) and high (Williams et al., 2005) attentional load manipulations. On the other hand, ignored fearful faces presented foveally lead to an increase of amygdala response under low-load (Anderson et al., 2003; Pessoa et al., 2005), but not high-load conditions (Pessoa et al., 2002, 2005).

The proposed interaction between the attentional load of a competing task and the stimulus location suggests that the amygdala may be especially sensitive to affective stimuli presented in the periphery in order to attract attention to important items that are not in the current focus of attention. Such a function would be supported by input from the magnocellular pathway, which produces rapid, transient, but coarse visual signals and is particularly sensitive to peripheral stimuli (Livingstone and Hubel, 1987, 1988; Bullier, 2001). Indeed, high spatial-frequency information in faces travels via parvocellular inputs into ventral cortical pathways and evokes responses in fusiform cortex, whereas the amygdala preferentially responds to low spatial-frequency information carried primarily by magnocellular visual pathways (Vuilleumier et al., 2003; Winston et al., 2003).

Although these findings support the hypothesis that the attentional load as well as the spatial position of the emotional stimuli are critical factors for the strength of amygdala activation by faces, no neuroimaging study to date varied both factors to directly test their interaction. Therefore, in the present study we investigated the effects of attentional load and stimulus location on the processing of emotional faces in the amygdala using functional magnetic resonance imaging (fMRI) in the same group of subjects. Pairs of stimuli depicting faces were presented at one of three eccentricities along the horizontal meridian: Adjacent to the central fixation point, on the edge of the screen and in between these two extreme positions. In addition, visual streams consisting of letters and digits were presented simultaneously in each visual field quadrant (Morawetz et al., 2007). During the attend-faces conditions, participants had to match either the gender or the valence of the facial expression. During the ignore-faces conditions, subjects had to deploy their attention to either one (low-load) or two streams (high-load) at opposite locations relative to the fixation point, performing a match/mismatch judgment. This experimental design allowed us to manipulate the attentional load, without changing the stimulus display and with identical decision types (match/mismatch judgment) for attend-faces and ignore-faces conditions. Unlike previous studies, we were able to directly test the interaction between attentional load and stimulus location in the visual field within the same group of participants.

With regard to the effects of attentional load on amygdala activity, we hypothesized to find (1) a reduced response during ignore-faces compared to attend-faces conditions. Concerning the effects of different spatial locations of the emotional stimuli on amygdala activity, we hypothesized to find (2) an enhanced amygdala response to centrally presented faces during the low, but not high attentional load condition, and (3) an increase in amygdala activity during both high and low attentional load conditions when the faces are presented more peripherally.

\section{MATERIALS AND METHODS SUBJECTS}

Fourteen right-handed, healthy adults (nine males) aged 20-34 years (mean \pm SD: $25.8 \pm 3.0$ years) with normal or corrected to normal vision were examined. All subjects gave written informed consent to participate in the study, which was approved by the local ethics committee.

Prior to the imaging sessions, participants completed the Positive and Negative Affective Schedule (PANAS; Watson et al., 1988) to assess different current emotional states and feelings as well as a self-report measure of attentional control, the Attentional Control Scale (ACS; Derryberry and Reed, 2002).

The PANAS includes 20 items that assess two orthogonal factors of affective state, positive affect and negative affect (Watson and Tellegen, 1985). Scores on each scale range from 10 to 50. On the PANAS, participants' positive affect scores ranged from 22 to 35 (mean \pm SD: $28.2 \pm 3.2$ ) and their negative affect scores from 10 to $17(12.8 \pm 2.5)$. These scores fall within the published norms (positive affect: $29.7 \pm 7.9$; negative affect: $14.8 \pm 5.4$; Watson et al., 1988).

The ACS consists of 20 items to assess individual differences in attentional skills related to voluntary executive functions. The scale measures a general capacity for attentional control, with correlated subfactors related to the ability to focus attention, to shift attention between tasks and to flexibly control thought (Derryberry and 
Reed, 2002). Scores on the ACS range from 20 to 80. Participants' scores ranged from 47 to $66(55.6 \pm 6.2)$, comparable to the results of Bishop et al. (2007).

Furthermore, subjects rated the valence and the level of arousal of all face stimuli using a computerized version of the SelfAssessment Manikin (SAM) scale (Bradley and Lang, 1994). The SAM is a self-administered scale with icons that indicate graphically how arousing and pleasant stimuli are. Participants rated each face picture on pleasantness (ranging from extremely pleasant to extremely unpleasant) and arousal (ranging from extremely aroused to extremely calm). The version of the SAM used in the present study included five icons that define a 9-point scale for each dimension with 9 representing a very pleasant and extremely exciting stimulus. The mean valence ratings for neutral $(4.2 \pm 0.4)$, happy $(6.6 \pm 0.8)$, and fearful $(2.9 \pm 0.6)$ stimuli showed that all faces were evaluated in accordance with the intended portrayed facial expression. The mean arousal ratings for fearful and happy faces indicated that these faces were neither perceived as very calm nor as extremely exciting (happy faces: $5.5 \pm 0.9$; fearful faces: $5.9 \pm 0.9)$, whereas neutral faces were rated as less arousing $(3.7 \pm 0.6)$.

\section{STIMULI}

The emotional stimuli consisted of grayscale face photographs of 70 individuals ( 35 male, 35 female), each portraying a happy, fearful, and neutral expression and were selected from the Karolinska Directed Emotional Faces collection (Lundqvist et al., 1998). All faces were presented within the same oval frame to avoid discrimination based on outer contours. As performance declines toward the visual field periphery in most tasks, when a constant stimulus size is used (Weymouth, 1958), we scaled the photographs by the human cortical magnification factor to activate an approximately equivalent portion of early visual cortex at all stimulated eccentricities (Rovamo and Virsu, 1979; Van Essen et al., 1984; Tolhurst and Ling, 1988). We scaled our pictures based on the equation $M^{-1}=(1+a E) \times M_{0}^{-1}$ (Rovamo and Virsu, 1979) with $E$ referring to eccentricity in degrees and $M_{0}$ representing the value of magnification.

Pairs of faces were presented along the horizontal meridian for $550 \mathrm{~ms}$ and then masked by images with the amplitude spectra but scrambled face spectra for $1050 \mathrm{~ms}$. Face stimuli centered $1.68^{\circ}$ from central fixation (central-position) had a height of $3^{\circ}$, pictures presented at a distance of $5.6^{\circ}$ from fixation (mid-position) were $5^{\circ}$ high and photographs at $11.25^{\circ}$ eccentricity (peripheralposition) measured $7^{\circ}$. The spatial positioning of the emotional faces remained the same during the experimental blocks, but was varied in between runs.

Additionally to the two faces, letters and digits were displayed in rapid serial visual presentation (RSVP). Four RSVP streams were presented simultaneously, one in each visual field quadrant $\left(8.5^{\circ}\right.$ diagonally from central fixation; Morawetz et al., 2007). The letter height subtended a visual angle of $2.3^{\circ}$ and each stimulus was shown for $183 \mathrm{~ms}$ (resulting in 110 letters and digits during one experimental block of $20 \mathrm{~s}$ ), which precluded deliberate saccades during their presentation and shifts of attention between the target locations (Reeves and Sperling, 1986; Weichselgartner and Sperling, 1987; Duncan et al., 1994; Peterson and Juola, 2000). The stimuli were presented consecutively without an interstimulus interval.
All visual stimuli were presented on LCD-goggles (Resonance Technology, Northridge, USA) using the stimulation software Presentation (Version 10.3, Neurobehavioral Systems, USA).

\section{EXPERIMENTAL PROCEDURE}

The experiment consisted of four conditions: Match digits low, Match digits high, Match gender, and Match emotion (Figure 1).

In ignore-faces trials (Match digits low and Match digits high), participants had to attend to the RSVP streams and perform two different tasks of low and high attentional load. In the Match digits low task (low-load condition, Figure 1A) subjects had to maintain fixation on the fixation cross, and monitor one peripheral stream of digits and letters (lower left) for a predefined target which appeared every 1800-2000 ms. The other RSVP streams served as distractors. Different digits were defined as targets, which were constant within a run but varied between runs. In the Match digits high task (high-load condition, Figure 1B) subjects had to monitor two peripheral streams in opposing visual field quadrants for the simultaneous appearance of digits and indicate via button press whether the digits were the same (match) or different (mismatch). Each experimental block of $20 \mathrm{~s}$ contained 11 target events, six match and five mismatch trials.

In attend-faces trials (Match gender and Match emotion), subjects had to judge on presented face stimuli with and without explicit focus on the emotional content. In the Match gender task (Figure 1C) subjects were instructed to maintain fixation on the fixation cross in the middle of the screen and indicate via button press whether the presented faces matched in gender or not. In the Match emotion task (Figure 1D) participants pressed a button to indicate whether two faces showed the same or different emotional expression (fearful, happy). All blocks of faces contained an equal number of males and females and an equal number of positive, negative and neutral expressions. Each experimental block of $20 \mathrm{~s}$ contained 11 target events, six match and five mismatch trials. During Match emotion mismatch trials consisted randomly of either a combination of two neutral faces, a neutral and an emotional face or two emotional faces of the opposite emotional target expression, e.g., if targets were fearful faces, then distractors were pairs of happy faces or pairs of a happy and a neutral face or two neutral faces. During Match gender emotional expressions of the faces were randomized within an experimental block.

To summarize, the stimulus display consisted of a fixation cross in the middle of the screen, a pair of faces followed by a mask stimulus at one of the three spatial locations (central-position, mid-position, peripheral-position) and the four RSVP streams (Figure 1E).

A block design was chosen to increase the functional signal-tonoise ratio in the amygdala as well as to make use of the advantages associated with it, such as the robustness of the results, increased statistical power and relatively large MR-signal changes relative to the baseline (Amaro and Barker, 2006). The four experimental conditions were separated from each other by a Fixation condition (20 s) containing an instruction display that indicated the type of task (4s). During the Fixation condition a black fixation cross was presented in the center of a white background. Experimental runs started with a fixation phase (20 s) and then altered between task (20 s) and Fixation condition (20 s) resulting in a block of 


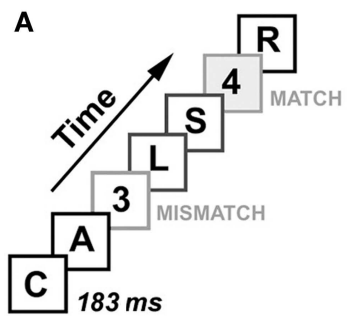

MATCH DIGITS LOW

C

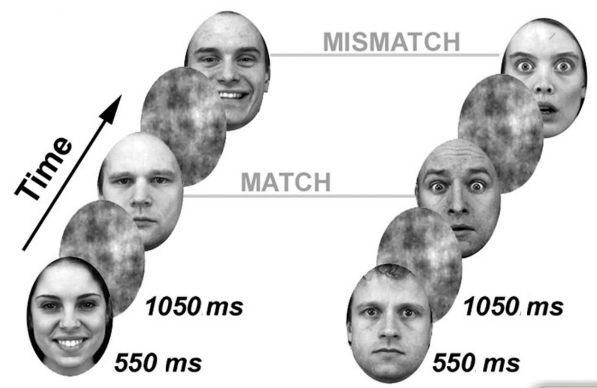

B

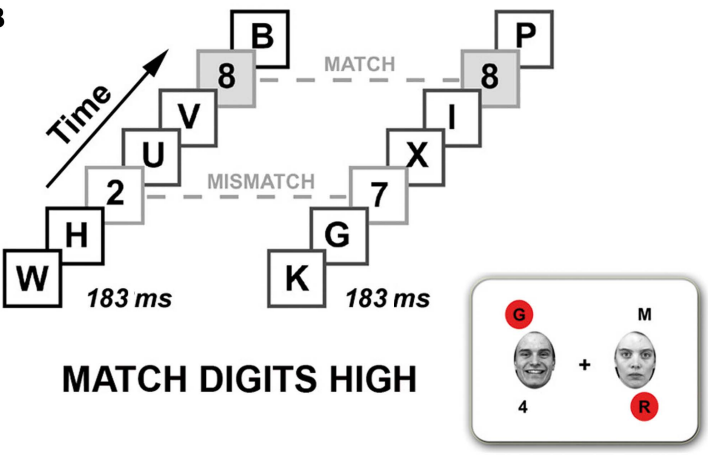

D

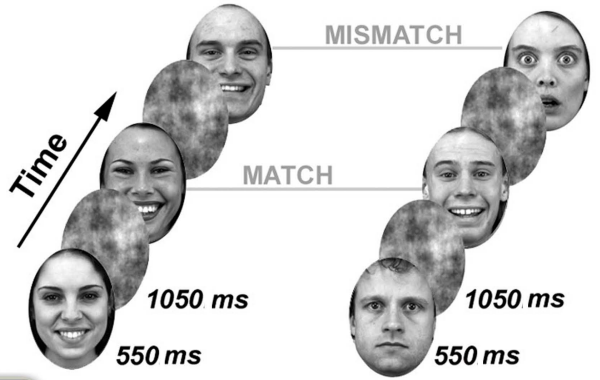

MATCH GENDER

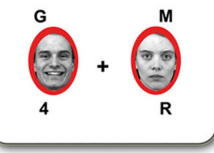

MATCH EMOTION

Task Relevance

E

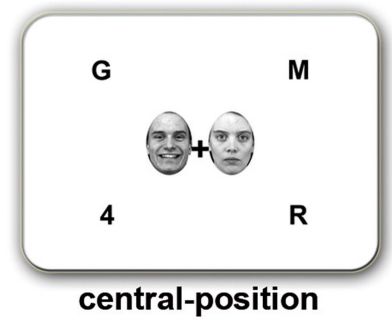

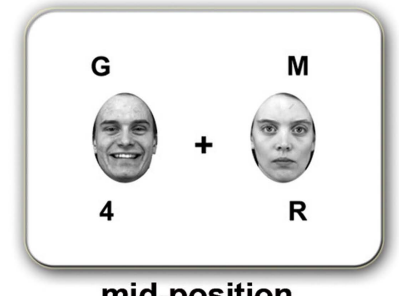

mid-position

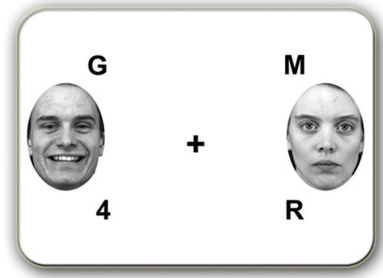

peripheral-position

$\mathbf{F}$
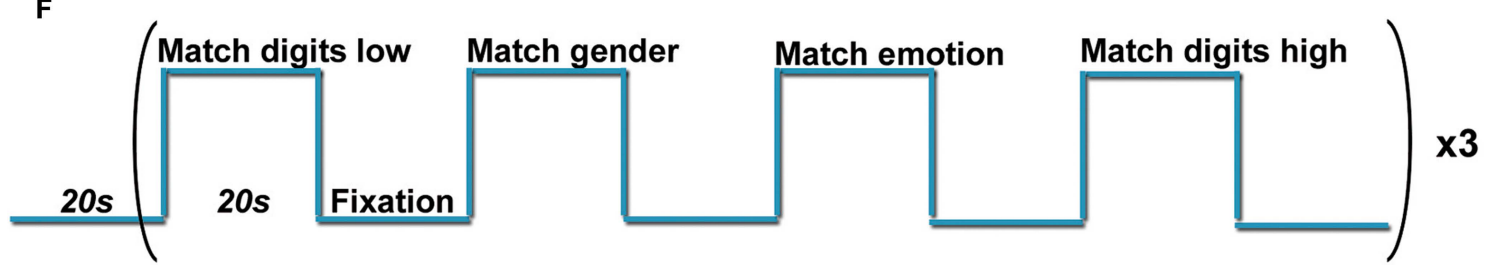

FIGURE 1 | Tasks and experimental design. The visual display consisted of four rapid serial visual presentation (RSVP) streams presented simultaneously in each visual field quadrant and a pair of faces presented horizontally. Task relevance is indicated in red. (A) Match digits low task. Subjects had to monitor the lower left stream for the appearance of digits and indicate via button press if the digits matched or mismatched the target. Distractor streams had to be ignored. (B) Match digits high task. The upper left and lower right RSVP streams were defined as target locations. Simultaneously appearing digits were considered as targets. Participants had to indicate via button press whether the digits were the same (match) or different (mismatch). (C) Match gender task. Subjects had to attend the faces and determine whether they match in gender (match) or not (mismatch). (D) Match emotion task. Subjects had to decide whether the facial expressions match (match) or not (mismatch). (E) Spatial location of the face stimuli. Pairs of faces were presented either in the central-, mid-, or peripheral-position. (F) The stimulation paradigm employed for functional imaging. One block consisted of four different tasks, each lasting 20 s, separated by a Fixation condition and repeated three times.
$160 \mathrm{~s}$, which was repeated three times (Figure 1F). Accordingly, one experimental run lasted $8 \mathrm{~min} 20 \mathrm{~s}$ and was repeated four times during one scan session. Tasks and blocks were presented in a pseudo-randomized order. Three scan sessions per subject were required to obtain the datasets for the three different spatial positions of the face stimuli in the visual field. Scan sessions were 
separated from each other by 1 week. The study was counterbalanced with respect to both task conditions (varied within runs) and eccentricity of the emotional stimuli (varied between runs, within and between scan sessions).

Participants had to respond as quickly and accurately as possible, by pressing one of two buttons on a fiber optic computer response device (Current Designs, Inc., Philadelphia, USA). Subjects were instructed that fixation of the central cross should be maintained throughout the experiment. Only the focus of attention alternated between face stimuli and RSVP streams. Before scanning subjects were trained on the different tasks in separate sessions. The adjusted frequency of correct answers ([\%correct/(\%correct + \%incorrect) $] \times 100$ ), omitting trial responses missed by the subject, was calculated for all four tasks for each participant in order to exclude subjects for whom the adjusted frequency of correct answers was not different from chance level. The behavioral data were analyzed using a $3 \times 4$ repeated measures ANOVA with location of the emotional stimuli and task as within-subject factors.

\section{IMAGE ACQUISITION}

Magnetic resonance imaging was performed at 3 Tesla (Magnetom Trio, Siemens Healthcare, Erlangen, Germany). Initially, a highresolution 3D T1-weighted dataset was acquired from each subject (176 sagittal sections, $1 \times 1 \times 1 \mathrm{~mm}^{3}$ ). For fMRI a T2*-weighted, gradient-echo echo planar imaging technique recording $22 \mathrm{sec}$ tions of $4 \mathrm{~mm}$ thickness oriented roughly parallel to the anterior and posterior commissure at an in-plane resolution of $2 \times 2 \mathrm{~mm}^{2}$ was used (repetition time $=2000 \mathrm{~ms}$; echo time $=36 \mathrm{~ms}$; fieldof-view $=192 \times 256 \mathrm{~mm}^{2}$ ). For each experimental run 250 whole brain volumes were recorded.

\section{IMAGE PROCESSING AND ANALYSIS}

Data were analyzed within the framework of a random effects general linear model using BrainVoyager QX 1.9 (Brain Innovation, Maastricht, The Netherlands). Preprocessing of fMRI data included 3D-motion-correction, temporal high pass filtering (3 cycles/run), linear trend removal, spatial smoothing (Gaussian smoothing kernel, $4 \mathrm{~mm}$ full width half maximum), and transformation into the space of Talairach and Tournoux (1988).

As the main goal of this study was to investigate the effect of attentional load and spatial location of the emotional stimuli on amygdala responses to unattended fearful and happy faces, we performed a region of interest (ROI) analysis of the amygdala. ROIs of the amygdalae were determined anatomically on a single subject level (mean coordinates: left: $x, y, z=-19 \pm 0.6,-5 \pm 0.7$, $-11 \pm 0.4 ;$ size $=552 \mathrm{~mm}^{3}$; right: $x, y, z=20 \pm 0.6,-5 \pm 0.7$, $-11 \pm 0.3$; size $=639 \mathrm{~mm}^{3}$; Figure $2 \mathrm{~A}$ ). The left and right amygdalae were identified by one of the authors (Carmen Morawetz) on the T1-weighted 3D dataset of each subject according to the protocol of Pruessner et al. (2000). First we determined the posterior end of the amygdala in the coronal plane, at the point where gray matter first started to appear superior to the alveus and laterally to the hippocampal head. We used the horizontal view to define the medial and lateral border. The medial border was identified by looking for the border of the ambient cistern in the posterosuperior section of the amygdala. The lateral border was determined by the lateral half of the semicircle drawn from the uncal
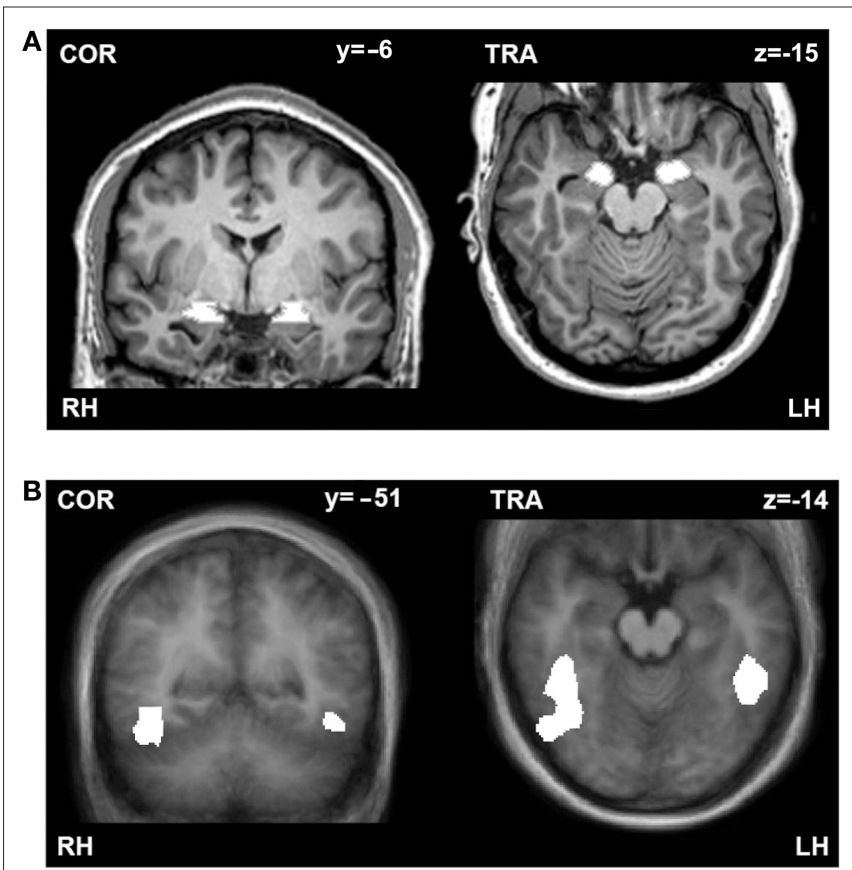

FIGURE 2 | (A) Anatomically defined example ROIs of the amygdalae. Coronal (left) and transversal (right) section through the amygdala of one subject. (B) Functionally defined ROls of the fusiform gyri. Coronal (left) and transversal (right) section through the fusiform gyrus displayed on an average brain of the whole group. ROls are indicated in white. $\mathrm{RH}$, right hemisphere; $\mathrm{LH}$, left hemisphere.

recess of the inferior horn of the lateral ventricle medially to the inferior horn of the lateral ventricle laterally. The inferior border was defined on coronal images. The anterior border was identified at the level of the closure of the lateral sulcus. This protocol allowed a consistent approach for the definition of the amygdala. The coordinates are consistent with the peaks of activation recently reported in a meta-analysis of 94 imaging studies $(x, y, z= \pm 22$, -6, -12; Costafreda et al., 2008).

In addition to the amygdala we investigated the fusiform gyri to verify the successful implementation of our attentional load manipulation paradigm as this region is known to be implicated in face processing (Kanwisher et al., 1997) and modulated by selective attention (Wojciulik et al., 1998; Reddy et al., 2007).

In three of our subjects we localized the fusiform gyrus in a separate fMRI experiment by presenting blocks of grayscale face or house images interleaved with Fourier images, which were all fit behind an oval mask. Each image was presented in the middle of the screen for $500 \mathrm{~ms}$ and each block lasted $18 \mathrm{~s}$. Contrasting the faces versus houses $(q($ FDR $)<0.001)$ resulted in functional ROIs of significantly activated voxels in the left $(x, y, z=-45,-44,-14$; size $\left.=1618 \mathrm{~mm}^{3}\right)$ and right $\left(x, y, z=41,-53,-13\right.$; size $\left.=6295 \mathrm{~mm}^{3}\right)$ fusiform gyrus (Figure 2B). The coordinates are consistent with the mean peaks of activation reported in the Brede Database (Nielsen, 2003) for these regions (mean coordinates: left: $x, y, z=-38 \pm 7$, $-57 \pm 12,-13 \pm 6$; right: $x, y, z=37 \pm 9 ;-52 \pm 16,-14 \pm 7$ ).

To investigate habituation effects in the amygdala and fusiform gyrus, we split the experimental blocks within one run into early and late blocks allowing us to probe how both attentional load of 
the tasks and spatial location of the faces depended on time. As one run consisted of 12 task blocks (each task was repeated three times in one run), the first block of each task condition within one run was assigned early, whereas the last run of each task condition was labeled late (blocks between early and late were grouped as intermediate).

The left and right ROIs of the amygdala were combined for further analyses. Before pooling the ROIs, we tested for laterality effects by applying a three-factors repeated measures 4 (task) by 3 (location) by 2 (side) ANOVA analysis, which included the "side" of the ROI (left or right) as additional factor. The results revealed no significant laterality effects in the amygdala $(F(1,13)=0.03$, $p=0.85$ ), which is in line with several meta-analysis (Zald, 2003; Costafreda et al., 2008; Sergerie et al., 2008).

The left and right fusiform gyrus ROIs were analyzed separately at first. The observed differential effects of the attentional load manipulation and the eccentricity of the emotional stimuli were more prominent in the right fusiform gyrus than in the left, which is in agreement with previous studies reporting an attentional modulation only in the right fusiform gyrus (Pessoa et al., 2002; Silvert et al., 2007). It is important to note that the left-visual-field superiority for faces has been attributed to a right hemispheric dominance for face perception (Sergent and Bindra, 1981; Rhodes, 1985; Yovel et al., 2008) and that this neural asymmetry in face processing is a stable individual characteristic (Yovel et al., 2008). Besides, there is abundant evidence suggesting strong functional differences between right and left fusiform gyrus (Kanwisher and Yovel, 2006; Dien, 2009). On the basis of all these reports supporting a lateralization of the fusiform gyrus regarding face processing, only the right fusiform gyrus ROI was considered as control region.

For the selected ROIs the estimated effects (beta values) of each factor-level combination for each subject were extracted. The obtained beta values were used as input for the second-level ANOVA $(3 \times 4 \times 3)$ analysis (two-sided) with location of the emotional stimuli, task, and time as within-subject factors. Finally, we computed the mean percent signal change averaged across participants for each experimental condition with respect to the spatial locations of the emotional stimuli. Differences were tested using paired $t$-tests.

\section{RESULTS}

\section{BEHAVIORAL DATA}

To assess behavioral performance, mean reaction times (RTs) for each experimental condition and each subject were computed. First, we examined task effects on RTs considering the spatial location of the emotional faces (Figure 3A).

The ANOVA analysis of RTs revealed a significant main effect of task $(F(3,39)=48.956, p<0.001)$, no significant main effect of location $(F(2,26)=0.218, p=0.804)$ as well as no significant interaction effect between task and location $(F(6,78)=1.402, p=0.213)$. During the ignore-faces conditions, participants responded significantly faster during Match digits low than Match digits high, when the unattended faces were presented in the central-position $(F(1,13)=11.9$, $p<0.001)$, mid-position $(F(1,13)=13.4, p<0.001)$, and peripheralposition $(F(1,13)=19.5, p<0.001)$. During the attend-faces conditions, subjects matched the expression significantly faster than the

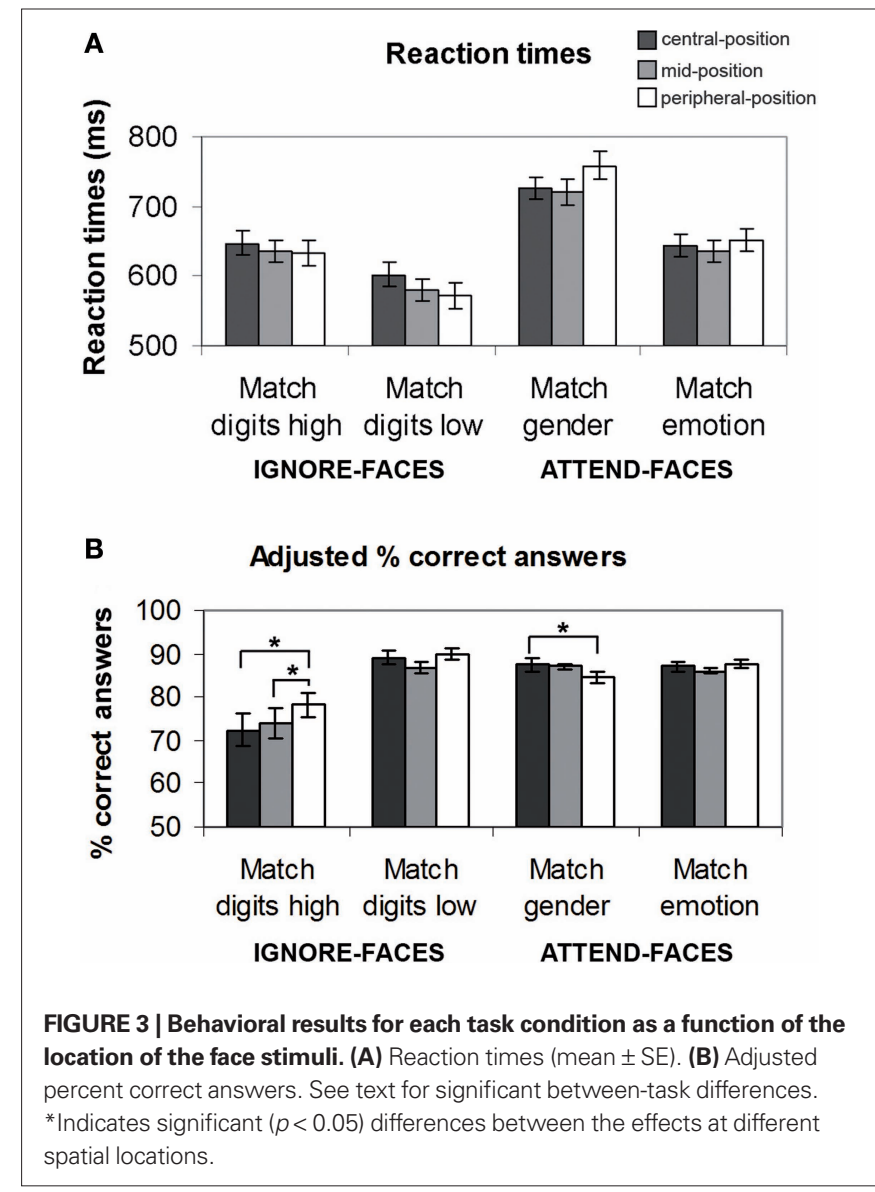

gender of the faces at all three spatial locations (central-position: $F(1,13)=65.5, p<0.001$; mid-position: $F(1,13)=73.8, p<0.001$; peripheral-position: $F(1,13)=141.2, p<0.001)$.

In a second step, the efficacy of the attentional load manipulation was tested by assessing the accuracy of the performance during the different experimental conditions (Figure 3B). Repeated measures ANOVA revealed a significant main effect of task $(F(3,39)=13.063, p<0.001)$, no significant main effect of location $(F(2,26)=1.631, p=0.215)$, but a significant interaction between task and location $(F(6,78)=4.655, p<0.001)$. As expected, mean task performance for all conditions was well above chance level $(p<0.001)$. Subjects were more accurate in Match digits low than in Match digits high, regardless of the face distractor positions (central-position: $F(1,13)=22.6, p<0.001$; mid-position: $F(1,13)=17.0, p<0.001$; peripheral-position: $F(1,13)=19.7, p<0.001)$. During attend-faces trials subjects performed significantly better in Match emotion than in Match gender only when the faces were presented in the peripheralposition $(F(1,13)=5.2, p<0.04)$. During Match gender performance was significantly higher during trials in which the faces were presented at the central-position compared to the peripheral-position $(F(1,13)=5.1, p=0.04)$. Interestingly, subjects were more accurate during Match digits high, when the faces were located in the peripheral-position rather than in the central-position $(F(1,13)=13.4, p<0.003)$ or in the mid-position $(F(1,13)=5.9, p<0.03)$. 
In between scan sessions no significant difference in task performance was observed for the attend-face trials, whereas during Match digits low and Match digits high, a significant increase in performance was obtained from the first scan session compared to the third scan session (Match digits low: $F(1,13)=7.0, p<0.02$; Match digits high: $F(1,13)=5.0, p<0.04)$.

\section{NEUROIMAGING DATA}

\section{Amygdala region of interest analysis}

In a first step we tested for habituation effects in the amygdala. Applying a three-factors repeated measures 4 (task) by 3 (location) by 3 (time) ANOVA analysis revealed no significant main effect of time $(F(2,26)=0.54, p=0.584)$ and no interaction effect between task and time $(F(6,78)=0.54, p=0.774)$ in the amygdala. Between location and time no significant interaction effect was found $(F(4,52)=1.14, p=0.345)$. Therefore, all blocks were grouped together regardless of the time of their presentation within a run and used for further analysis.

In a second step we investigated the main and interaction effects of attentional load and spatial location of the emotional stimuli. A significant main effect of task was observed in amygdala $(F(3,39)=49.94, p<0.001)$, whereas the main effect of location did not reach significance $(F(2,26)=1.41, p=0.26)$. Furthermore, no significant interaction effect between task and location was observed in the amygdala $(F(6,78)=0.69, p=0.65)$.

Effect of attentional load. Testing hypothesis 1 favoring the concept of attentional load dependent amygdala activation, we examined the role of cognitive and emotional modulation by comparing responses evoked by the different task conditions relative to Fixation. According to hypothesis 1, the amygdala would be preferentially activated during the attend-faces conditions (Match emotion and Match gender). The results revealed a strong dependence of amygdala responses on the allocation of attention as the two ignore-faces conditions (Match digits low and Match digits high) caused a significant suppression of responses compared to the two attend-faces tasks resulting in a significant difference $(F(1,13)=83.0, p<0.001$; Figure 4A; all statistically significant comparisons between conditions are listed in Table 1).

Effect of stimulus eccentricity. According to hypothesis 2, stimulus location should affect the signal change in the amygdala during Match digits low compared to Match digits high in the central-position. However, the response in the amygdala was not influenced by location of the face stimuli during these two task conditions, when the faces were presented in the central-position (Figure $4 B$ ). The largest decreases in signal change were found during Match digits low and Match digits high with presentation of the face stimuli in the mid-position (Figure 4B).

Hypothesis 3 is based on the assumption that peripherally presented stimuli compared to centrally ones are associated with an increase in amygdala activity during Match digits low as well as Match digits high. In contrast to this hypothesis, no significant difference in signal change was observed in the amygdala contrasting the two attentional load conditions in the peripheral-position with the central-position $(F(1,13)=0.31, p=0.582)$. Only the contrast between the mid-position and the central-position resulted in differential amygdala responses, demonstrating a stronger decrease in signal change during Match digits low when the face stimuli are presented in the mid-position $(F(1,13)=5.98, p=0.02$; Figure 4B; all statistically significant comparisons between conditions are listed in Table 1).

\section{Fusiform gyrus region of interest analysis}

First, we looked for habituation effects and applied a three-factors repeated measures 4 (task) by 3 (location) by 3 (time) ANOVA analysis. The results revealed a significant main effect of time in the right fusiform gyrus $(F(2,26)=34.92, p<0.001)$. Testing for an interaction effect between time and location resulted in no significant effect $(F(4,52)=0.77, p=0.547)$, whereas between task and time an interaction effect $(F(6,78)=3.55, p=0.003)$ was observed in the right fusiform gyrus.

As the ANOVA analysis clearly demonstrated a habituation effect within the right fusiform gyrus and an interaction between task and time, we determined the underlying characteristics of these effects. The results showed that the signal change decreased over time. The early blocks of the attend-faces and ignore-faces conditions compared to the late blocks were associated with a stronger response in the right fusiform gyrus (attend-faces: central-position: $F(1,27)=10.5, p=0.003$; mid-position: $F(1,27)=10.1, p=0.004$; peripheral-position: $F(1,27)=7.8, p=0.009$; ignore-faces: centralposition: $F(1,27)=7.2, p=0.012$; mid-position: $F(1,27)=8.1$, $p=0.008)$. Therefore, only the early blocks of each task condition were included for further analysis.

Secondly, we tested for main effects of task and location and interaction effects of these two factors. The analysis revealed a significant main effect of task $(F(3,39)=7.3, p<0.001)$ and location $(F(2,26)=4.5, p=0.02)$ in the right fusiform gyrus. No significant interaction effect between task and location was observed $(F(6,78)=0.6, p=0.669)$.

In the next step we investigated the nature of the main effect of the attentional load and spatial location of the emotional stimuli on right fusiform gyrus activity. Overall, an increase in response was found during attend-faces as well as ignore-faces conditions in the right fusiform gyrus compared to the Fixation condition.

Effect of attentional load. A significant effect of attentional load was observed in the right fusiform gyrus as an increase in signal change was found in the attend-faces compared to the ignore-faces conditions $((\mathrm{F} 1,13)=11.7, p=0.002$; Figure $5 \mathrm{~A}$; all statistically significant comparisons between conditions are listed in Table 2).

Significantly stronger responses were observed in Match emotion compared to Match digits low and Match digits high. Matching gender was associated with significantly higher signal changes in the right fusiform gyrus in contrast to the high attentional load task, when the faces were presented in the central- and peripheral-position.

The contrast between the ignore-faces conditions revealed stronger responses for the low attentional load task in the right fusiform gyrus, when the face stimuli were presented in the central- and peripheral-position. Within the attend-faces conditions a significant difference was found: Matching the expression of the faces compared to matching the gender resulted in higher responses at all three stimulus locations. 


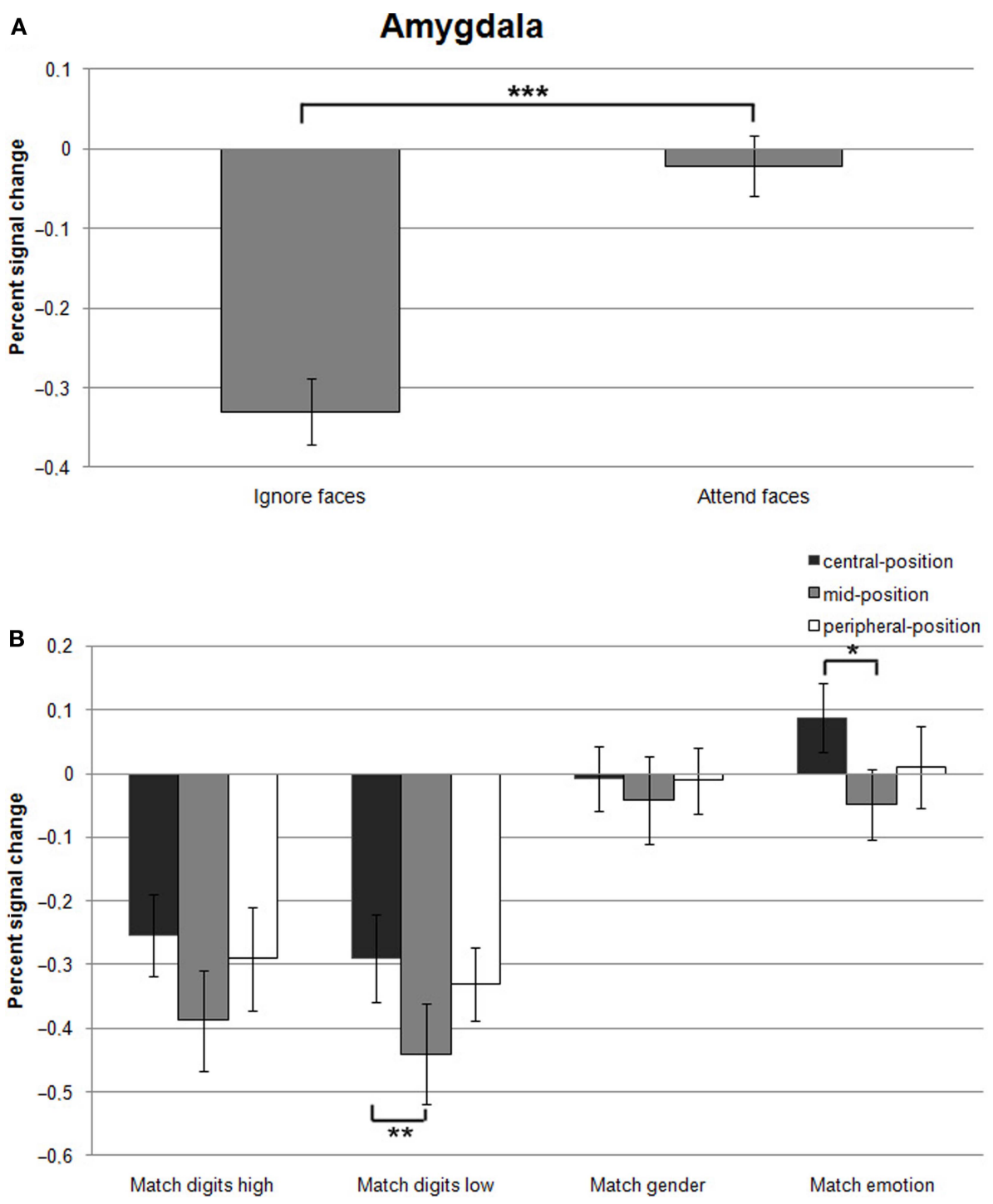

FIGURE 4 | (A) Mean percent signal changes ( \pm SE) relative to the Fixation condition in the amygdala during Ignore-faces and Attend-faces conditions.

***Indicates significant difference between conditions at $p<0.001$. (B) Mean percent signal changes $( \pm \mathrm{SE})$ relative to the Fixation condition in the amygdala during the Match digits high, Match digits low, Match gender, and Match emotion task as a function of the spatial location of the face stimuli. ${ }^{*}$ and ${ }^{* *}$ indicate significant $(p<0.05$ and $p<0.01)$ differences between the effects at different spatial locations. See text and Table $\mathbf{1}$ for between-task comparisons.
Effect of stimulus eccentricity. Within all task conditions, an increase of activation in the right fusiform gyrus was observed with decreasing eccentricity (Figure 5B; all statistically significant comparisons between conditions are listed in Table 2). The location of the faces affected responses in the right fusiform gyrus during all task conditions by producing a significant increase in activity when the emotional stimuli were located in the central- rather than in the peripheral-position.

During the high-load condition, enhanced responses were found, when the ignored stimuli were positioned in the central- and mid-position compared to the peripheral one. When subjects directed their attention to the faces by matching the gender in the central- compared to the mid-position, a significant increase in activity was observed in the right fusiform gyrus. Facial expression matching also induced an increase in activity when the attended stimuli were presented in the mid- rather than in the peripheral-position.

\section{DISCUSSION}

In the present study, we succeeded in approaching the issue of attention related activation of the amygdala in regard to the processing of emotional facial expressions: Our experimental design allowed a direct assessment not only of the impact of attentional load, but also the effect of spatial location on the emotional stimuli and the interaction between both factors within the same group of subjects. Clearly favoring the concept of the attentional load theory, our results revealed a strong modulation of signal changes in the amygdala by the availability 
Table 1 | Lists of all statistically significant comparisons between task conditions.

\begin{tabular}{|c|c|c|c|}
\hline Contrast & Position $^{a}$ & $F(1,27)$ & $p$-Value \\
\hline \multicolumn{4}{|c|}{ EFFECT OF ATTENTIONAL LOAD IN THE AMYGDALA } \\
\hline Attend-faces > & Central & 44.6 & 0.001 \\
\hline \multirow[t]{3}{*}{ ignore-faces } & Mid & 126.3 & 0.001 \\
\hline & Peripheral & 63.1 & 0.001 \\
\hline & Position $^{a}$ & $F(1,13)$ & $p$-Value \\
\hline Match emotion> & Central & 93.23 & 0.001 \\
\hline \multirow[t]{2}{*}{ match digits low } & Mid & 89.82 & 0.001 \\
\hline & Peripheral & 28.62 & 0.001 \\
\hline Match emotion> & Central & 93.23 & 0.001 \\
\hline \multirow[t]{2}{*}{ match digits high } & Mid & 50.97 & 0.001 \\
\hline & Peripheral & 24.80 & 0.001 \\
\hline Match gender> & Central & 27.78 & 0.001 \\
\hline \multirow[t]{2}{*}{ match digits low } & Mid & 76.28 & 0.001 \\
\hline & Peripheral & 38.19 & 0.001 \\
\hline Match gender> & Central & 7.52 & 0.017 \\
\hline \multirow[t]{2}{*}{ match digits high } & Mid & 31.58 & 0.001 \\
\hline & Peripheral & 22.64 & 0.001 \\
\hline $\begin{array}{l}\text { Match emotion> } \\
\text { match gender }\end{array}$ & Central & 4.87 & 0.046 \\
\hline \multicolumn{4}{|c|}{ EFFECT OF STIMULUS ECCENTRICITY INTHE AMYGDALA } \\
\hline Match digits low > fixation & Central > mid & 7.81 & 0.015 \\
\hline Match emotion > fixation & Central > mid & 4.97 & 0.044 \\
\hline
\end{tabular}

Only statistically significant comparisons are listed in the table.

a Position of the face stimuli in the visual field.

of attentional resources. Furthermore, it could be shown that the responses in the amygdala were not consistently affected by the spatial positions of the faces. Therefore, the findings suggest that the processing of emotional stimuli is strongly modulated by selective attention but is independent of stimulus eccentricity. Furthermore, no significant relationship between the different tasks (and associated attentional load) and stimulus location could be demonstrated.

The behavioral results support the successful implementation of the attentional load manipulation in the present study. Our findings showed that subjects responded faster and more accurately during Match digits low than Match digits high indicating that the split of attention among two spatial positions in opposing quadrants of the visual field induces an increase in task difficulty and is more attentionally demanding (high attentional load), in line with previous results (Morawetz et al., 2007). To keep performance of foveal and peripheral face recognition at a comparable level, image size was increased with eccentricity. Indeed, during the attend-faces conditions mean task performance was retained with increasing eccentricity. Furthermore, there was no difference between Match emotion and Match gender. These results suggest that difficulty was approximately equivalent in each task, when response accuracy alone is considered. However, the analysis of the RTs revealed that expression match- ing was significantly faster than gender classifications, suggesting that for our stimuli expression was more easily discriminable than gender.

\section{AMYGDALA}

The present findings of a lack of differential signal changes in the amygdala during the different task conditions are in agreement with the proposal that in areas implicated in emotional processing, such as the amygdala, cerebral blood flow decreases during the performance of some attentionally demanding cognitive tasks (Drevets and Raichle, 1998). Demanding cognitive tasks that withdraw attention from the emotional stimuli are associated with an active suppression of amygdala responses elicited during a default state, which is suspended during many types of cognitive processing (Shulman et al., 1997; Raichle et al., 2001). Thus, the decreased and minimal activity during the active tasks may reflect the absence of processes that occur during neutral or passive conditions. This implies that the preferential activation of the amygdala by emotional facial expressions may not only depend on attention to the faces, but instead may require that no suppression processes are active at the time of stimulus presentation. Therefore, we argue that the visual discrimination tasks in the present study have been cognitively demanding to such an extent that limbic regions have been inhibited.

A possible reason for the failure in activating the amygdala might have been the way the emotional stimuli have been presented. During many trials the presented face pairs were not congruent regarding their emotional valence causing an inhomogeneity within the emotional stimuli. This absence of congruence in the facial expressions possibly reduced the responses in the amygdala. In order to address concerns about the experimental design we conducted a control experiment in a subgroup of three subjects to explore the impact of actively judging on or passively viewing of faces on the amygdala response. They had to either perform the Match emotion task or to view the face stimuli passively as a second condition (the RSVP streams were removed from the visual display; the timing was the same as in the main experiment and blocks were interleaved by a fixation condition). Passive viewing of the face pairs compared to matching the expression of the faces resulted in a significant increase of signal change in the left $(t=7.3, p<0.001)$ and right $(t=13.9, p<0.001)$ amygdala. Thus, we suggest that the observed lack of signal increase in the amygdala is not attributable to the experimental design, but reflects the influence of an active task on signal changes in this limbic region. Indeed, deactivation of the amygdala has been observed in several tasks that involve higher cognitive processing (Drevets and Raichle, 1995; Shulman et al., 1997) and in active visual tasks (i.e., attentionally demanding tasks in which subjects make decisions regarding various visual stimuli) relative to passive viewing tasks (Shulman et al., 1997). Furthermore, a recent meta-analysis of 385 imaging studies showed that task instructions involving a form of attentional processing reduce amygdala activation compared to the passive processing of emotional stimuli (Costafreda et al., 2008).

In regard to hypothesis 1, our findings of a variable suppression of amygdala response to attended and unattended faces are in contrast to studies that failed to find effects of attentional 

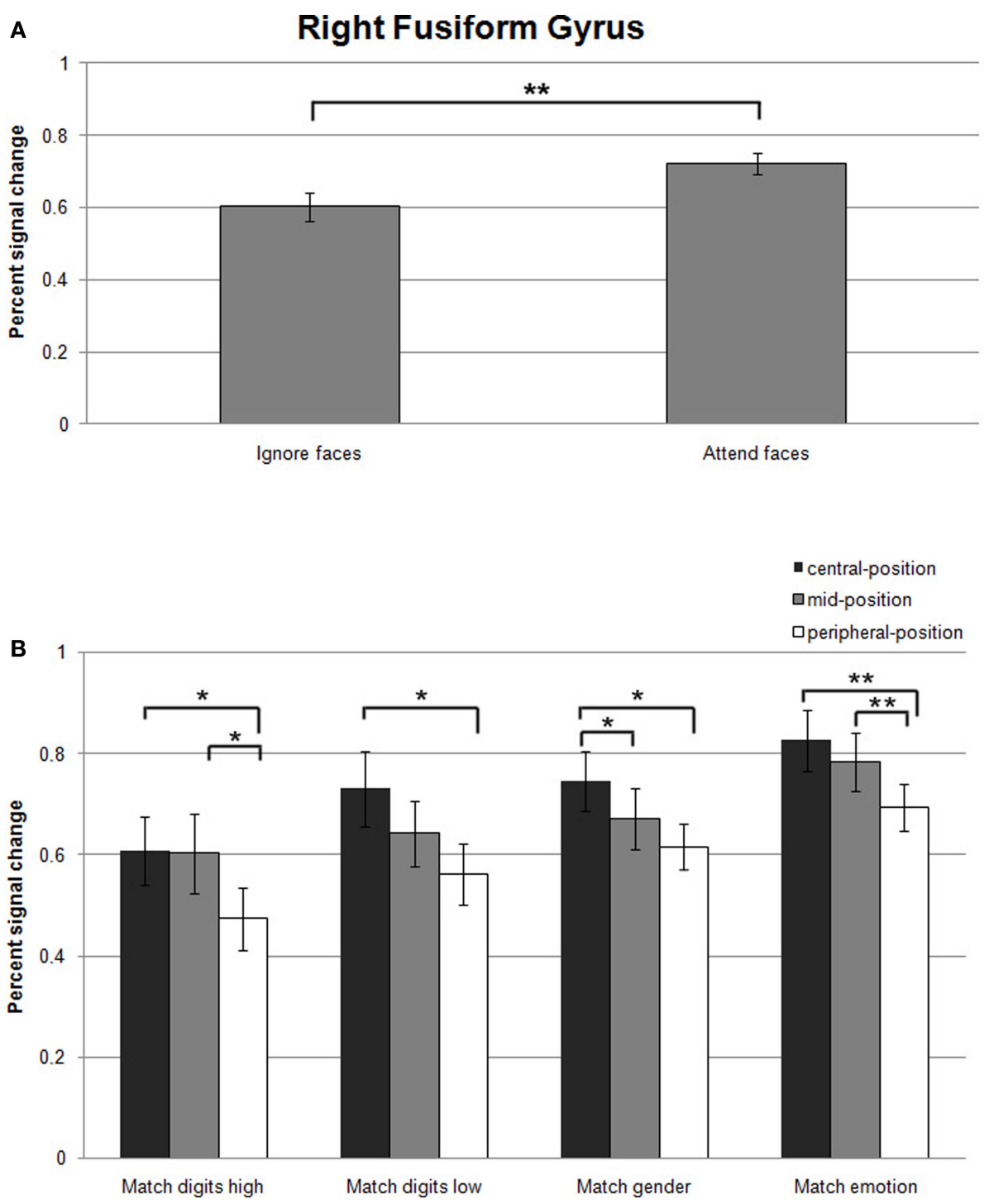

FIGURE 5 | (A) Mean percent signal changes ( $\mathbf{A S E}$ ) relative to the Fixation condition in the right fusiform gyrus during Ignore-faces and Attend-faces conditions. ${ }^{*}$ Indicates significant difference between conditions at $p<0.01$. (B) Mean percent signal changes $( \pm S E$ ) relative to the Fixation condition in the right fusiform gyrus during the Match digits high, Match digits low, Match gender, and Match emotion task as a function of the spatial location of the face stimuli. * and ${ }^{*}$ indicate significant $(p<0.05$ and $p<0.01)$ differences between the effects at different spatial locations. See text and Table 2 for between-task comparisons. load manipulations on amygdala responses to emotional stimuli (Vuilleumier et al., 2001; Anderson et al., 2003; Williams et al., 2005). Although a decrease in neuronal activity in the amygdala during the ignore-faces conditions was found, the obtained differences between the tasks indicate that the attentional load manipulations still had an effect. During the explicit processing of the faces (Match gender and Match emotion) only weak signal changes in the amygdala were observed in contrast to when the faces were ignored and attention was directed to the letters and digits (Match digits low and Match digits high). Our results are therefore in line with previous studies demonstrating discriminative responses of the amygdala to attended and unattended faces (Pessoa et al., 2002, 2005; Bishop et al., 2007; Mitchell et al., 2007; Silvert et al., 2007) supporting hypothesis 1 . Similarly, a decrease in amygdala activity has been documented when faces of different emotional valence were unattended (Pessoa et al., 2002) and when a demanding task of high attentional load was performed (Pessoa et al., 2005; Bishop et al., 2007; Mitchell et al., 2007). In addition, a decreased amygdala response was found during high-load attentional tasks (without emotional faces being present in the visual display) compared to fixation (Pessoa et al., 2005) and compared to a task specific baseline (Hsu and Pessoa, 2007). The attentional load manipulation of the tasks used to direct attention away from the faces in the aforementioned studies is one of the main differences in contrast to previous studies supporting the notion of an automatic responsitivity of the amygdala to fearful faces. In these studies the attentional load of the different task conditions was not varied. The subjects had to direct their attention either to 
Table 2 | Lists of all statistically significant comparisons between task conditions.

\begin{tabular}{|c|c|c|c|}
\hline Contrast & Position $^{a}$ & $F(1,27)$ & $p$-Value \\
\hline \multicolumn{4}{|c|}{ EFFECT OF ATTENTIONAL LOAD IN THE RIGHT FUSIFORM GYRUS } \\
\hline Attend-faces > & Central & 6.9 & 0.014 \\
\hline \multirow[t]{3}{*}{ ignore-faces } & Mid & 9.18 & 0.005 \\
\hline & Peripheral & 10.43 & 0.003 \\
\hline & Positiona & $F(1,13)$ & $p$-Value \\
\hline Match emotion > & Central & 4.9 & 0.044 \\
\hline \multirow{2}{*}{ match digits low } & Mid & 11.1 & 0.005 \\
\hline & Peripheral & 5.4 & 0.036 \\
\hline Match emotion> & Central & 10.2 & 0.007 \\
\hline \multirow{2}{*}{ match digits high } & Mid & 16.4 & 0.001 \\
\hline & Peripheral & 12.8 & 0.003 \\
\hline \multirow{3}{*}{$\begin{array}{l}\text { Match gender > } \\
\text { match digits high }\end{array}$} & Central & 5.2 & 0.039 \\
\hline & Mid & & \\
\hline & Peripheral & 6.7 & 0.022 \\
\hline \multirow{3}{*}{$\begin{array}{l}\text { Match digits low > } \\
\text { match digits high }\end{array}$} & Central & 6.1 & 0.027 \\
\hline & Mid & & \\
\hline & Peripheral & 6.3 & 0.025 \\
\hline \multirow{3}{*}{$\begin{array}{l}\text { Match emotion> } \\
\text { match gender }\end{array}$} & Central & 4.9 & 0.044 \\
\hline & Mid & 8.3 & 0.013 \\
\hline & Peripheral & 5.5 & 0.035 \\
\hline \multicolumn{4}{|c|}{ EFFECT OF STIMULUS ECCENTRICITY IN THE RIGHT FUSIFORM GYRUS } \\
\hline \multirow[t]{2}{*}{ Match digits high > fixation } & Central > peripheral & 7.3 & 0.018 \\
\hline & Mid > peripheral & 6.2 & 0.027 \\
\hline Match digits low > fixation & Central > peripheral & 6.8 & 0.021 \\
\hline \multirow[t]{2}{*}{ Match gender > fixation } & Central > mid & 12.4 & 0.004 \\
\hline & Central > peripheral & 24 & 0.001 \\
\hline \multirow[t]{2}{*}{ Match emotion > fixation } & Central > peripheral & 12.4 & 0.004 \\
\hline & Mid > peripheral & 9.2 & 0.01 \\
\hline
\end{tabular}

Only statistically significant comparisons are listed in the table.

${ }^{a}$ Position of the face stimuli in the visual field.

faces or houses matching their identity (Vuilleumier et al., 2001; Williams et al., 2005) or perform a male/female judgment on the faces and an inside/outside judgment on the houses (Anderson et al., 2003). It has been proposed that complete suppression of amygdala responses to unattended faces may occur only, when the competing tasks exhaust the attentional resources available leaving almost no capacities for processing task-irrelevant stimuli (Pessoa et al., 2005). Thus, as outlined above, we suggest that in our study both attentional load manipulations were very effective in diverting attention from the faces thereby inducing a particularly strong suppression of neuronal activity in the amygdala. This impact on the amygdala response is supported by the fact that Match digits low as well as Match digits high resulted in a similar decrease in activation even though the behavioral results indicated that Match digits high demanded higher attentional load than Match digits low. Therefore, no differences in amygdala response to centrally presented faces during the low compared to the high attentional load condition were observed, as has been proposed in hypothesis 2 .
One of the main goals of the present study was to investigate the effect of stimulus eccentricity on amygdala responses (hypothesis 3). The previously discussed studies (Pessoa et al., 2002, 2005; Anderson et al., 2003; Bishop et al., 2007; Mitchell et al., 2007) presented the emotional stimuli foveally. It has therefore been difficult to assess the proposal that the spatial location of the emotional stimuli constitutes an important factor in determining amygdala activation (Palermo and Rhodes, 2007). To date, only one fMRI study has investigated the impact of attentional load on the processing of peripherally presented faces (Silvert et al., 2007). These authors showed that differential amygdala responses to emotional versus neutral unattended faces vanished with increasing attentional load, supporting a resource-dependent activation model of the amygdala responsiveness. However, in contrast to our results, no complete suppression of amygdala activation was observed, when the faces were unattended in the high attentional load task. In our paradigm, presenting faces at three different eccentricities in the visual field to investigate the effect of stimulus location on amygdala responses, we did not observe a significantly higher response when stimuli were presented at the peripheral-position for our four respective task conditions. This is in disagreement with hypothesis 3 regarding the influence of stimulus location on amygdala activity, thus not supporting the idea that the amygdala may be especially sensitive to affective stimuli presented in the periphery.

An important issue that needs to be addressed are methodological differences between the various studies. For data analysis we used an ROI-based approach, which is statistically powerful because only a small number of a priori specified ROIs are analyzed reducing the severity of correction for multiple tests and supporting spatial correspondence mapping across subjects (Poldrack, 2007). However, it has the disadvantage of a reduced possibility of detecting small foci of activation by narrowly focusing on preselected regions (Poldrack, 2007).

Regarding the experimental design, we used a block design in the present study, while previous imaging reports used either mixed block/event-related designs (Pessoa et al., 2002; Bishop et al., 2007), event-related designs (Vuilleumier et al., 2001; Anderson et al., 2003; Mitchell et al., 2007), or blocked designs (Pessoa et al., 2005; Silvert et al., 2007). These differences between existing studies make a direct comparison difficult. While experiments using a block design in general feature an increased statistical power and more robust results, transient activation changes at block onset and/or offset might be missed (Amaro and Barker, 2006). However, we expected to constantly affect the activation level in the amygdala by the attentional load manipulation for the whole duration of our task blocks. Another possible drawback of block designs is their susceptibility to habituation effects. To avoid habituation effects in our study, task condition blocks were interleaved by fixation blocks and the design was fully counterbalanced with regard to task conditions (within runs) and location of the face stimuli (between runs). However, previous studies reported that the amygdala response habituates (i.e., decreases over time) to repeated presentations of human facial expressions (Breiter et al., 1996; Wright et al., 2001; Britton et al., 2008). Thus, the habituation might result in attenuated differences between experimental conditions in this limbic region. Such findings prompted us to test for habituation effects in the amygdala. Our findings differ from previously reported data (Phillips et al., 2001; Fischer et al., 2003; Williams 
et al., 2004) as we did not observe a rapid habituation of the amygdala to repeatedly presented emotional stimuli thereby disproving the idea that the signal decrease could be attributed to habituation. Supporting our results, a recent study (Haas et al., 2008) also failed to find any evidence for amygdala habituation. It has been suggested that these inconsistencies likely reflect methodological differences in the temporal parameters as the studies varied in the length of blocks and experimental runs. It has been proposed that the amygdala does not exhibit habituation when shorter (e.g., $18 \mathrm{~s}$ ) blocks are used (Haas et al., 2008), which is consistent with our data and design (one block lasted 20 s).

\section{FUSIFORM GYRUS}

In line with previous neuroimaging studies (Hsu and Pessoa, 2007; Britton et al., 2008) a time-related differential activation of the right fusiform gyrus has been observed, prompting us to only include the early blocks of each run for the evaluation of attentional load and spatial location effects in order to minimize habituation effects. This control region revealed increased activity during all task conditions, while the attend-faces compared to the ignore-faces condition resulted in stronger activation. Moreover, an increase of activation in the right fusiform gyrus was observed with decreasing distance between the faces for all tasks, indicating the successful implementation of our experimental paradigm.

The involvement of the fusiform gyrus in the processing of faces has been established by numerous imaging studies demonstrating greater activity to faces than to nonsense or non-face stimuli (Sergent et al., 1992; Haxby et al., 1994, 1999; Puce et al., 1996; Kanwisher et al., 1997; McCarthy et al., 1997; Halgren et al., 1999; Ishai et al., 1999; Hoffman and Haxby, 2000). In line with these previous results we observed an increase in activity in the right fusiform gyrus during all tasks conditions. As the visual display contained face stimuli in all task blocks, we also found increased responses in the fusiform gyrus when the faces were ignored. This is consistent with the results of Silvert et al. (2007).

Furthermore, the analysis revealed a differential activity induced by the different tasks thereby pointing toward an attentional modulation within this ROI. In agreement with other fMRI studies we

\section{REFERENCES}

Adolphs, R. (2002). Neural systems for recognizing emotion. Curr. Opin. Neurobiol. 12, 169-177.

Amaral, D. G., Behniea, H., and Kelly, J. L. (2003). Topographic organization of projections from the amygdala to the visual cortex in the macaque monkey. Neuroscience 118, 1099-1120.

Amaro, E. Jr., and Barker, G. J. (2006). Study design in $\mathrm{AMRI}$ : basic principles. Brain Cogn. 60, 220-232.

Anderson, A. K., Christoff, K., Panitz, D., De Rosa, E., and Gabrieli, J. D. (2003). Neural correlates of the automatic processing of threat facial signals. J. Neurosci. 23, 5627-5633.

Anderson, A. K., and Phelps, E. A. (2001). Lesions of the human amygdala impair enhanced perception of emotionally salient events. Nature 411, 305-309.
Bishop, S. J., Jenkins, R., and Lawrence, A. D. (2007). Neural processing of fearful faces: effects of anxiety are gated by perceptual capacity limitations. Cereb. Cortex 17, 1595-1603.

Bradley, M. M., and Lang, P. J. (1994). Measuring emotion: the SelfAssessment Manikin and the semantic differential. J. Behav. Ther. Exp. Psychiatry 25, 49-59.

Breiter, H. C., Etcoff, N. L., Whalen, P. J., Kennedy, W. A., Rauch, S. L., Buckner, R. L., Strauss, M. M., Hyman, S. E., and Rosen, B. R. (1996). Response and habituation of the human amygdala during visual processing of facial expression. Neuron 17, 875-887.

Britton, J. C., Shin, L. M., Barrett, L. F., Rauch, S. L., and Wright, C. I. (2008). Amygdala and fusiform gyrus temporal dynamics: responses to negative

observed a significant increase in activity in the right fusiform gyrus when face stimuli were presented inside the focus of attention (Wojciulik et al., 1998; O’Craven et al., 1999; Vuilleumier et al., 2001; Pessoa et al., 2002; Williams et al., 2005; Reddy et al., 2007; Silvert et al., 2007).

The spatial location of the face stimuli also influenced the responses in the right fusiform gyrus. Here, activation was significantly stronger in response to central compared to peripheral face stimuli, in line with previous studies (Levy et al., 2001; Liu and Ioannides, 2006).

Testing different spatial locations of emotional stimuli in the visual field in relation to demanding cognitive tasks simultaneously withdrawing attention from the same, this fMRI study clearly demonstrates (1) that cognitively demanding tasks involving active attentional processing lead to an inhibition of amygdala responses indicating an attention related resource-dependence of face encoding processes and (2) that the spatial location of emotional stimuli does not affect amygdala activity.

In summary, our results suggest that the processing of emotional information in the amygdala is governed by top-down processes involved in selective attention. This is reminiscent of the strong dependency of responses across visual cortex on attentional allocation to the respective visual stimuli. The observed modulation of amygdala responses by attention will contribute to the generation of an integrated saliency map (Treue, 2003) in which the strength of representation of an emotional stimulus is a combination of its emotional valence ("salience") with the behavioral context in which attention can be allocated or diverted from the stimulus.

\section{ACKNOWLEDGMENTS}

Carmen Morawetz was supported by the European graduate school Neuroplasticity: From Molecules to Systems of the Deutsche Forschungsgemeinschaft (GRK 632). Carmen Morawetz, Juergen Baudewig, and Peter Dechent were supported by the Volkswagen Foundation. Stefan Treue and Carmen Morawetz were supported by the German Ministry for Education and Science Grant BMBF 01GQ0433 to the Bernstein Center for Computational Neuroscience, Goettingen.

facial expressions. BMCNeurosci. 9, 44 . doi: 10.1186/1471-2202-1189-1144.

Bullier, J. (2001). Integrated model of visual processing. Brain Res. Brain Res. Rev. 36, 96-107.

Compton, R. J. (2003). The interface between emotion and attention: a review of evidence from psychology and neuroscience. Behav. Cogn. Neurosci. Rev. 2, 115-129.

Costafreda, S. G., Brammer, M. J., David, A. S., and Fu, C. H. (2008). Predictors of amygdala activation during the processing of emotional stimuli: a meta-analysis of 385 PET and fMRI studies. Brain Res. Rev. 58, 57-70.

Derryberry, D., and Reed, M. A. (2002). Anxiety-related attentional biases and their regulation by attentional control. J. Abnorm. Psychol. 111, 225-236.
Desimone, R., and Duncan,J.(1995).Neural mechanisms of selective visual attention. Annu. Rev. Neurosci. 18, 193-222.

Dien, J. (2009). A tale of two recognition systems: implications of the fusiform face area and the visual word form area for lateralized object recognition models. Neuropsychologia 47, 1-16.

Drevets, W. C., and Raichle, M. E. (1995). "PET imaging studies of human emotional disorders," in The Cognitive Neurosciences, ed. M. S. Gazzaniga (Cambridge, MA: MIT Press), 1153-1164.

Drevets, W. C., and Raichle, M. E. (1998). Reciprocal suppression of regional cerebral blood flow during emotional versus higher cognitive processes: implications for interactions between emotion and cognition. Cogn. Emot. 12, 353-385. 
Duncan, J., Ward, R., and Shapiro, K. (1994). Direct measurement of attentional dwell time in human vision. Nature 369, 313-315.

Fischer, H., Wright, C. I., Whalen, P. J., McInerney, S. C., Shin, L. M., and Rauch, S. L. (2003). Brain habituation during repeated exposure to fearful and neutral faces: a functional MRI study. Brain Res. Bull. 59, 387-392.

Haas, B. W., Constable, R. T., and Canli, T. (2008). Functional magnetic resonance imaging of temporally distinct responses to emotional facial expressions. Soc. Neurosci. 4, 121-134.

Halgren, E., Dale, A. M., Sereno, M. I., Tootell, R. B., Marinkovic, K., and Rosen, B. R. (1999). Location of human face-selective cortex with respect to retinotopic areas. Hum. Brain Mapp. 7, 29-37.

Haxby, J. V., Horwitz, B., Ungerleider, L. G., Maisog, J. M., Pietrini, P., and Grady, C. L. (1994). The functional organization of human extrastriate cortex: a PET-rCBF study of selective attention to faces and locations. $J$. Neurosci. 14(Pt 1), 6336-6353.

Haxby, J. V., Ungerleider, L. G., Clark, V. P., Schouten, J. L., Hoffman, E. A., and Martin, A. (1999). The effect of face inversion on activity in human neural systems for face and object perception. Neuron 22, 189-199.

Hoffman, E. A., and Haxby, J. V. (2000). Distinct representations of eye gaze and identity in the distributed human neural system for face perception. Nat. Neurosci. 3, 80-84.

Hsu, S. M., and Pessoa, L. (2007). Dissociable effects of bottom-up and top-down factors on the processing of unattended fearful faces. Neuropsychologia 45, 3075-3086.

Ishai, A., Ungerleider, L. G., Martin, A., Schouten, J. L., and Haxby, J.V. (1999). Distributed representation of objects in the human ventral visual pathway. Proc. Natl. Acad. Sci. U.S.A. 96, 9379-9384.

Kanwisher, N., McDermott, J., and Chun, M. M. (1997). The fusiform face area: a module in human extrastriate cortex specialized for face perception. $J$. Neurosci. 17, 4302-4311.

Kanwisher, N., and Yovel, G. (2006). The fusiform face area: a cortical region specialized for the perception of faces. Philos. Trans. R Soc. Lond., B., Biol. Sci. 361, 2109-2128.

Kastner, S., and Ungerleider, L. G. (2000). Mechanisms of visual attention in the human cortex. Annu. Rev. Neurosci. 23, 315-341.

Killgore, W. D., and Yurgelun-Todd, D. A. (2004). Activation of the amygdala and anterior cingulate during nonconscious processing of sad versus happy faces. Neuroimage 21, 1215-1223.
Lavie, N. (1995). Perceptual load as a necessary condition for selective attention. J. Exp. Psychol. Hum. Percept. Perform. 21, 451-468.

Lavie, N. (2005). Distracted and confused? Selective attention under load. Trends Cogn. Sci. 9, 75-82.

LeDoux, J.E. (1996). The Emotional Brain: The Mysterious Underspinnings of Emotional Life. New York: Touchstone Press.

LeDoux, J. E., Sakaguchi, A., and Reis, D. J. (1984). Subcortical efferent projections of the medial geniculate nucleus mediate emotional responses conditioned to acoustic stimuli. J. Neurosci. 4, 683-698.

Levy, I., Hasson, U., Avidan, G., Hendler, T., and Malach, R. (2001). Centerperiphery organization of human object areas. Nat. Neurosci. 4, 533-539.

Liddell, B. J., Brown, K. J., Kemp, A. H., Barton, M. J., Das, P., Peduto, A., Gordon, E., and Williams, L. M.(2005). A direct brainstem-amygdala-cortical 'alarm' system for subliminal signals of fear. Neuroimage 24, 235-243.

Liu, L., and Ioannides, A. A. (2006). Spatiotemporal dynamics and connectivity pattern differences between centrally and peripherally presented faces. Neuroimage 31, 1726-1740.

Livingstone, M., and Hubel, D. (1988). Segregation of form, color, movement, and depth: anatomy, physiology, and perception. Science 240, 740-749.

Livingstone, M. S., and Hubel, D. H. (1987). Psychophysical evidence for separate channels for the perception of form, color, movement, and depth. J. Neurosci. 7, 3416-3468.

Lundqvist, D., Flykt, A., and Ohman, A. (1998). The Karolinska Directed Emotional Faces (KDEF). Stockholm: Karolinska Institute.

McCarthy, G., Puce, A., Gore, J. C., and Allison, T. (1997). Face-specific processing in the human fusiform gyrus. J. Cogn. Neurosci. 9, 605-610.

Mitchell, D. G. V., Nakic, M., Fridberg, D., Kamel, N., Pine, D. S., and Blair, R. J. R. (2007). The impact of processing load on emotion. Neuroimage 34, 1299-1309.

Morawetz, C., Holz, P., Baudewig, J., Treue, S., and Dechent, P. (2007). Split of attentional resources in human visual cortex. Vis. Neurosci. 24, 817-826.

Morris, J.S., Friston, K. J., Buchel, C., Frith, C. D., Young, A. W., Calder, A. J., and Dolan, R. J. (1998a). A neuromodulatory role for the human amygdala in processing emotional facial expressions. Brain 121(Pt 1), 47-57.

Morris, J. S., Ohman, A., and Dolan, R. J. (1998b). Conscious and unconscious emotional learning in the human amygdala. Nature 393, 467-470.
Morris, J. S., Ohman, A., and Dolan, R. J. (1999). A subcortical pathway to the right amygdala mediating "unseen" fear. Proc. Natl. Acad. Sci. U.S.A. 96, 1680-1685.

Nielsen, A. F. (2003). The Brede database: a small database for functional neuroimaging. Presented at the 9th International Conference on Functional Mapping of the Human Brain, June 19-22, New York.

O'Craven, K. M., Downing, P. E., and Kanwisher, N. (1999). fMRI evidence for objects as the units of attentional selection. Nature 401, 584-587.

Öhman, A. (2002). Automaticity and the amygdala: nonconscious responses to emotional faces. Curr. Dir. Psychol. Sci. $11,62-66$.

Öhman,A.(2005). Therole of the amygdala in human fear: automatic detection of threat. Psychoneuroendocrinology 30, 953-958.

Öhman, A., Carlsson, K., Lundquist, D., and Ingvar, M. (2007). On the unconscious subcortical origin of human fear. Physiol. Behav. 92, 180-185.

Palermo, R., and Rhodes, G. (2007). Are you always on my mind? A review of how face perception and attention interact. Neuropsychologia 45, 75-92.

Pessoa, L. (2005). To what extent are emotional visual stimuli processed without attention and awareness? Curr. Opin. Neurobiol. 15, 188-196.

Pessoa, L., McKenna, M., Gutierrez, E. and Ungerleider, L. G. (2002). Neural processing of emotional faces requires attention. Proc. Natl. Acad. Sci. U.S.A. 99, 11458-11463.

Pessoa, L., Padmala, S., and Morland, T. (2005). Fate of unattended fearful faces in the amygdala is determined by both attentional resources and cognitive modulation. Neuroimage 28, 249-255.

Peterson, M. S., and Juola, J. F. (2000) Evidence for distinct attentional bottlenecks in attention switching and attentional blink tasks. J. Gen. Psychol. 127, 6-26.

Phan, K. L., Wager, T., Taylor, S. F., and Liberzon, I. (2002). Functional neuroanatomy of emotion: a meta-analysis of emotion activation studies in PET and fMRI. Neuroimage 16, 331-348.

Phelps, E. A. (2006). Emotion and cognition: insights from studies of the human amygdala. Annu. Rev. Psychol. 57, 27-53.

Phelps, E. A., and LeDoux, J. E. (2005). Contributions of the amygdala to emotion processing: from animal models to human behavior. Neuron 48, 175-187.

Phillips, M. L., Medford, N., Young, A. W., Williams, L., Williams, S. C., and Bullmore, E. T. (2001). Time courses of left and right amygdalar responses to fearful facial expressions. Hum. Brain Mapp. 12, 193-202.

Poldrack, R. A. (2007). Region of interest analysis for fMRI. Soc. Cogn. Affect. Neurosci. 2, 67-70.

Pruessner, J. C., Li, L. M., Serles, W., Pruessner, M., Collins, D. L., Kabani, N., Lupien, S., and Evans, A. C. (2000). Volumetry of hippocampus and amygdala with high-resolution MRI and three-dimensional analysis software: minimizing the discrepancies between laboratories. Cereb. Cortex 10, 433-442.

Puce, A., Allison, T., Asgari, M., Gore, J. C., and McCarthy, G. (1996). Differential sensitivity of human visual cortex to faces, letterstrings, and textures: a functional magnetic resonance imaging study. J. Neurosci. 16, 5205-5215.

Raichle, M. E., MacLeod, A. M., Snyder, A. Z., Powers, W. J., Gusnard, D. A., and Shulman, G. L. (2001). A default mode of brain function. Proc. Natl. Acad. Sci. U.S.A. 98, 676-682.

Reddy, L., Moradi, F., and Koch, C. (2007). Top-down biases win against focal attention in the fusiform face area. Neuroimage 38, 730-739.

Reeves, A., and Sperling, G. (1986). Attention gating in short-term visual memory. Psychol. Rev. 93, 180-206.

Rhodes, G. (1985). Lateralized processes in face recognition. Br. J. Psychol. 76, 249-271.

Rovamo, J., and Virsu, V. (1979). An estimation and application of the human cortical magnification factor. Exp. Brain Res. 37, 495-510.

Sergent, J., and Bindra, D. (1981). Differential hemispheric processing of faces: methodological considerations and reinterpretation. Psychol. Bull. 89, 541-554.

Sergent, J., Ohta, S., and MacDonald, B. (1992). Functional neuroanatomy of face and object processing. A positron emission tomography study. Brain 115, 15-36.

Sergerie, K., Chochol, C., and Armony, J. L. (2008). The role of the amygdala in emotional processing: a quantitative meta-analysis of functional neuroimaging studies. Neurosci. Biobehav. Rev. 32, 811-830.

Shulman, G. L., Corbetta, M., Buckner, R. L., Fiez, J. A., Miezen, F. M., Raichle, M. E., and Petersen, S. E. (1997). Common blood flow changes across visual tasks: II. Decreases in cerebral cortex. J. Cogn. Neurosci. 9, 647-662.

Silvert, L., Lepsien, J., Fragopanagos, N., Goolsby, B., Kiss, M., Taylor, J. G., Raymond, J. E., Shapiro, K. L., Eimer, M., and Nobre, A.C. (2007). Influence of attentional demands on the processing of emotional facial expressions in the amygdala. Neuroimage 38, 357-366. 
Talairach, J., and Tournoux, P. (1988). Co-planar Stereotaxic Atlas of the Human Brain. New York: Thieme.

Tolhurst, D. J., and Ling, L. (1988). Magnification factors and the organization of the human striate cortex. Hum. Neurobiol. 6, 247-254.

Treue, S. (2003). Visual attention: the where, what, how and why of saliency. Curr. Opin. Neurobiol. 13, 428-432.

Van Essen, D. C., Newsome, W. T., and Maunsell, J.H. (1984). The visual field representation in striate cortex of the macaque monkey: asymmetries, anisotropies, and individual variability. Vision Res. 24, 429-448.

Vuilleumier, P.,Armony, J.L., Driver, J., and Dolan, R. J. (2001). Effects of attention and emotion on face processing in the human brain: an event-related fMRI study. Neuron 30, 829-841.

Vuilleumier, P., Armony, J. L., Driver, J., and Dolan, R. J. (2003). Distinct spatial frequency sensitivities for processing faces and emotional expressions. Nat. Neurosci. 6, 624-631.

Vuilleumier,P., Richardson, M.P.,Armony, J. L., Driver, J., and Dolan, R. J. (2004). Distant influences of amygdala lesion on visual cortical activation during emotional face processing. Nat. Neurosci. 7, 1271-1278.

Watson, D., Clark, L. A., and Tellegen, A. (1988). Development and validation of brief measures of positive and negative affect: the PANAS scales. J. Pers. Soc. Psychol. 54, 1063-1070.

Watson, D., and Tellegen, A. (1985). Toward a consensual structure of mood. Psychol. Bull. 98, 219-235.

Weichselgartner, E., and Sperling, G. (1987). Dynamics of automatic and controlled visual attention. Science 238, 778-780.

Weymouth, F. W. (1958). Visual sensory units and the minimal angle of resolution. Am. J. Ophthalmol. 46(Pt 2), 102-113.

Whalen, P. J., Rauch, S. L., Etcoff, N. L., McInerney, S. C., Lee, M. B., and Jenike, M. A. (1998). Masked presentations of emotional facial expressions modulate amygdala activity without explicit knowledge. J. Neurosci. 18, 411-418.

Williams, L. M., Brown, K. J., Das, P., Boucsein, W., Sokolov, E. N., Brammer, M. J., Olivieri, G., Peduto, A., and Gordon, E. (2004). The dynamics of cortico-amygdala and autonomic activity over the experimental time course of fear perception. Brain Res. Cogn. Brain Res. 21, 114-123.

Williams, M. A., McGlone, F., Abbott, D. F., and Mattingley, J. B. (2005). Differential amygdala responses to happy and fearful facial expressions depend on selective attention. Neuroimage 24, 417-425.

Winston, J. S., Vuilleumier, P., and Dolan, R. J. (2003). Effects of low-spatial frequency components of fearful faces on fusiform cortex activity. Curr. Biol. 13, 1824-1829.

Wojciulik, E., Kanwisher, N., and Driver, J. (1998). Covert visual attention modulates face-specific activity in the human fusiform gyrus: fMRI study. J. Neurophysiol. 79, 1574-1578.

Wright, C. I., Fischer, H., Whalen, P. J., McInerney, S. C., Shin, L. M., and Rauch, S. L. (2001). Differential prefrontal cortex and amygdala habituation to repeatedly presented emotional stimuli. Neuroreport 12, 379-383.

Yovel, G., Tambini, A., and Brandman, T. (2008). The asymmetry of the fusiform face area is a stable individual characteristic that underlies the left-visual-field superiority for faces. Neuropsychologia 46, 3061-3068.

Zald, D. H. (2003). The human amygdala and the emotional evaluation of sensory stimuli. Brain Res. Brain Res. Rev. 41, 88-123.

Conflict of Interest Statement: The authors declare that the research was conducted in the absence of any commercial or financial relationships that could be construed as a potential conflict of interest.

Received: 04 August 2010; accepted: 22 November 2010; published online: 03 December 2010.

Citation: Morawetz C, Baudewig J, Treue $S$ and Dechent P (2010) Diverting attention suppresses human amygdala responses to faces. Front. Hum. Neurosci. 4:226. doi: 10.3389/fnhum.2010.00226

Copyright (C) 2010 Morawetz, Baudewig, Treue and Dechent. This is an open-access article subject to an exclusive license agreement between the authors and the Frontiers Research Foundation, which permits unrestricted use, distribution, and reproduction in any medium, provided the original authors and source are credited. 